\title{
DETERMINANTES SETORIAIS E SISTÊMICOS DAS EXPORTAÇÕES DE CARNE SUÍNA DOS ESTADOS UNIDOS: ANÁLISE COM QUEBRAS ESTRUTURAIS ${ }^{1}$
}

\section{SECTORAL AND SYSTEMIC DETERMINANTS OF PORK EXPORTS FROM UNITED STATES: ANALYSIS WITH STRUCTURAL BREAKS}

\author{
Tiane Alves Rocha Gastardelo ${ }^{2}$ \\ Laércio Juarez Melz ${ }^{3}$ \\ Pascoal José Marion Filho ${ }^{4}$ \\ Ariel Lopes Torres 5
}

\begin{abstract}
RESUMO
O objetivo do artigo foi verificar alguns dos determinantes para o desempenho das exportações dos Estados Unidos, utilizando quebras estruturais para localizar mudanças nos impactos destes determinantes. Para isso, foram coletadas séries trimestrais e aplicado o método de mínimos quadrados com quebras estruturais. Duas quebras estruturais foram localizadas, resultando em três regimes. O primeiro período (1990T1-1999T4) foi um período de com maior impacto de variáveis sistêmicas (PIB e inflação), no qual o país buscava ganhos de eficiência produtiva e maior competitividade na Cadeia de Valor Global. O segundo período (2000T1-2008T3) com maior impacto do PIB, inflação, taxa de juros e abates de suínos, já com a eficiência garantida por um sistema de contratos com coordenação vertical intensa. Um terceiro período (2008T4-2014T2) mais sensível às variáveis, tanto setoriais quanto sistêmicas, resultante da crise de 2008 além dos surtos de H1N1.
\end{abstract}

Palavras chave: Suinocultura, Cadeia de Valor Global, Competitividade.

\section{ABSTRACT}

The objective of this paper was to investigate some of the determinants for the United States pork exports performance by using structural breaks to locate changes in the impact of these determinants. For that, were collected quarterly time series and applied the method of least squares with structural breaks. Two structural breaks were located, resulting in three regimes. The first period (1990T11999T4) was a period with significant impact systemic variables (GDP and inflation), in which the country was seeking productive efficiency gains and improving competitiveness in the Global Value Chain. The second period (2000T1-2008T3) with the significant impact of GDP, inflation, interest rate and pig slaughtering, with efficiency guaranteed by a system of contracts with intense vertical coordination. A third period (2008T4-2014T2) more sensitive to variables changes, both sectoral and systemic, resulting from the 2008 crisis in addition to the H1N1 outbreak.

Keywords: Swine production, Global Value Chain, Competitiveness.

\section{INTRODUÇÃO}

A carne suína é a mais consumida no mundo, mesmo sofrendo pelas restrições devido aos hábitos, proibições religiosas e dogmáticas (GERVASIO, 2013) e, ainda, a despeito da crença de que carne suína é prejudicial à saúde, é uma carne magra e contém nutrientes

\footnotetext{
${ }^{1}$ Pesquisa financiada com recursos da Fundação de Amparo à Pesquisa do Estado de Mato Grosso - FAPEMAT.

${ }^{2}$ Mestranda em Administração (UFSM).

${ }^{3}$ Doutorando em Administração (UFSM), Professor do Departamento de Ciências Contábeis da UNEMAT, Bolsista FAPEMAT.

${ }^{4}$ Doutor em Economia Aplicada (USP). Professor do Doutorado em Administração da UFSM.

${ }^{5}$ Doutorando em Ciência Política (Uerj/Unemat), Professor do Departamento de Ciências Contábeis da UNEMAT, Bolsista FAPEMAT.
} 
semelhantes aos das demais carnes. Entre 1995 e 2012, o cenário mundial foi marcado por mudanças na composição dos principais exportadores. A Dinamarca, que era o maior exportador em 1995, com 18,4\% do mercado, teve decrescente participação, deixando seu posto para os Estados Unidos, em 2008, e para Alemanha em 2009. Esta passou a ser a maior exportadora mundial de carne suína, mantendo-se na posição até 2011 , com $15,9 \%$ do mercado. Estados Unidos apresentaram participação crescente no cenário, como segundo no ranking, entre 2008 e 2011 (14,6\%). O Brasil era o $9^{\circ}$ colocado no ranking em 1995, com $0,8 \%$ de participação. Sua maior participação aconteceu em 2002, com 6,9\% do mercado. Em 2011 o país participava de 4,5\% das exportações mundiais. Comparando o crescimento percentual de participação, o Brasil teve 464,9\% de crescimento, Alemanha 319,3\%, Estados Unidos $131,5 \%$ e Dinamarca -41,2\%. Isso demonstra que, no mercado de carne suína ainda há potencial de exportação para o Brasil (FAO, 2014).

O objetivo do artigo é verificar quais os determinantes das exportações de carne suína dos Estados Unidos para o resto do mundo entre 1990 e 2014. Para isso foram utilizadas séries temporais trimestrais. O método de quebras estruturais múltiplas foi utilizado para determinar as mudanças na dinâmica entre as variáveis.

\section{O MERCADO DA CARNE SUÍNA NO MUNDO E NOS ESTADOS UNIDOS}

No cenário mundial de carnes suínas destacam-se Alemanha, Estados Unidos, Dinamarca, Espanha, Canadá Holanda, Bélgica, França e Brasil. Estes países foram os nove maiores exportadores mundiais em 2011, segundo dados da Food and Agricultural Organization of the United Nations (FAO). É possível verificar que as exportações alemãs, em 1995, eram 4,75 vezes maiores que as brasileiras. Países como Dinamarca, Holanda, França e Canadá exportavam 23, 20, 10 e 7 vezes mais toneladas de carne suína que o Brasil. Em 2011 as exportações do Brasil passaram a ser quase iguais. O crescimento do Brasil como player no mercado internacional aconteceu a partir de 2002, quando experimentou um acréscimo de $75 \%$ nas suas exportações do produto (FAO, 2014). 
Tabela 1 - Exportações de carne suína dos nove maiores players, 1995-2011, em milhares de toneladas.

\begin{tabular}{cccccccccc}
\hline Ano & Alemanha & EUA & Dinamarca & Espanha & Canadá & Holanda & Bélgica & França & Brasil \\
\hline 1995 & 179,4 & 297,6 & 872,8 & 124,9 & 271,3 & 777,0 & & 393,0 & 37,8 \\
2000 & 398,3 & 543,6 & $1.092,0$ & 356,0 & 487,7 & 679,7 & 610,8 & 497,7 & 132,7 \\
2005 & 913,3 & 938,0 & $1.240,4$ & 633,1 & 811,3 & 721,5 & 631,0 & 507,3 & 686,4 \\
2006 & $1.023,1$ & $1.034,6$ & $1.251,8$ & 639,0 & 812,9 & 697,0 & 651,8 & 503,9 & 563,4 \\
2007 & $1.254,8$ & $1.115,3$ & $1.255,3$ & 700,2 & 771,9 & 780,5 & 691,4 & 644,2 & 666,2 \\
2008 & $1.508,8$ & $1.628,1$ & $1.243,3$ & 868,7 & 844,0 & 801,6 & 714,6 & 557,8 & 597,6 \\
2009 & $1.658,4$ & $1.448,1$ & $1.188,5$ & 937,8 & 840,3 & 786,0 & 735,8 & 530,5 & 637,4 \\
2010 & $1.763,8$ & $1.440,0$ & $1.249,1$ & 957,4 & 866,7 & 829,4 & 747,0 & 549,8 & 574,6 \\
2011 & $1.927,9$ & $1.766,0$ & $1.315,0$ & $1.045,7$ & 901,0 & 865,7 & 747,7 & 560,1 & 547,0 \\
\hline
\end{tabular}

Fonte: FAO (2014).

Os principais destinos da carne suína dos Estados Unidos entre 1990 e 2014 foram Canadá, China, Japão, Coréia do Sul e México. As exportações para estes países vêm crescendo continuamente, acelerando o ritmo de exportações para o Canadá e para a China, após 2009. Juntos, estes países importaram 77,75\% das carnes exportadas pelos EUA em 2014 (USDA, 2015a).

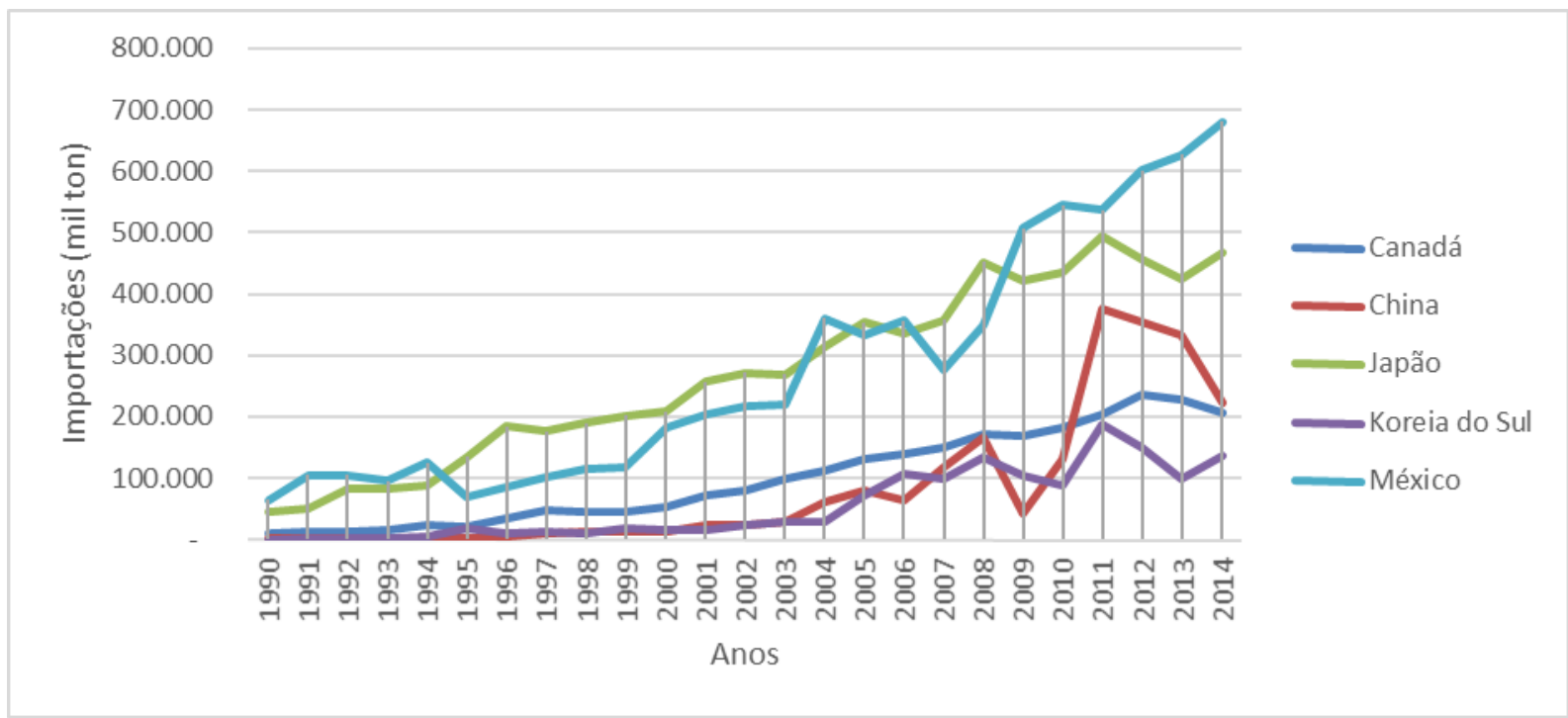

Fonte: USDA (2015b)

Figura 1 - Evolução das quantidades importadas pelos principais importadores de carnes dos Estados Unidos no período de 1990 a 2014, em mil toneladas

A tendência de crescimento foi acompanhada pela maior coordernação vertical executada a partir de contratos, nos quais grandes empresas são proprietárias dos suínos e contratam os suinocultores para criar os animais (LOWE; GEREFFI, 2008). Esta tendência de 
aumento dos contratos trouxe consigo o aumento expressivo da escala produtiva dos suinocultores e um aumento da especialização em terminação dos leitões, principalmente após 1999 (KEY; MCBRIDE, 2007).

\section{MÉTODO}

O modelo teórico que guia o presente artigo pode ser visto na figura 2. Neste modelo, as cadeias produtivas, neste caso da carne suína, fazem parte de um ambiente competitivo global, uma Cadeia de Valor Global (GEREFFI; HUMPHREY; STURGEON, 2005), e sofrem interferência de alguns determinantes setoriais e outros sistêmicos. Os determinantes setoriais são relacionados com a própria cadeia produtiva, como insumos (milho, soja, genética, etc) e os determinantes sistêmicos são relacionados com o ambiente econômico e institucional (PIB, inflação, taxas de juros, taxas de câmbio, etc.).

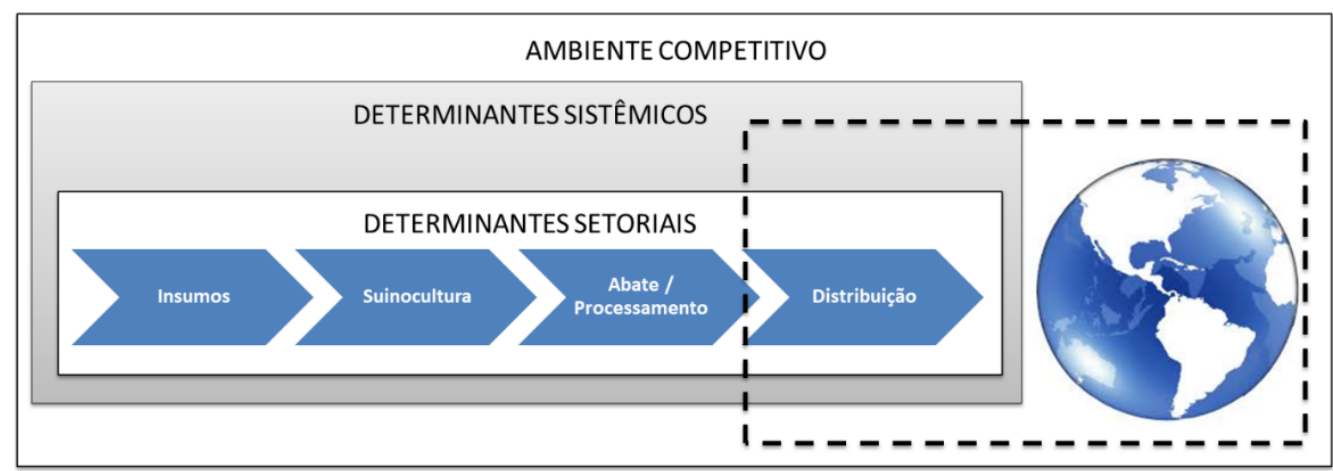

Figura 2 - Modelo teórico e foco do trabalho

Apesar de ser possível determinar a competitividade a partir de pesquisas qualitativas (BATALHA; SOUZA FILHO, 2009), neste artigo os resultados obtidos por meio de variáveis quantitativas, séries temporais, que podem ajudar a explicar os aumentos ou reduções nas exportações do país em foco, os Estados Unidos. O processamento será feito com uso de regressão por mínimos quadrados com quebras estruturais, explicado na próxima seção.

\subsection{Mínimos quadrados com quebras estruturais}

O modelo de Mínimos Quadrados com Quebras Estruturais, sugerido por Bai e Perron $(1998,2003)$ foi escolhido porque admite que as relações entre as variáveis independentes e a dependente são dinâmicas ao longo do tempo. O modelo utiliza um algoritmo de programação dinâmica no qual são estimados modelos com períodos diferentes, minimizando a soma dos quadrados dos resíduos. A regressão pode apresentar m quebras, sendo: 


$$
y_{t}=x_{t}^{\prime} \beta+z_{t}^{\prime} \delta_{j}+u_{t} \quad t=T_{j-1}+1, \ldots, T_{j}
$$

Para $\mathrm{j}=1, \ldots, \mathrm{m}+1$. Neste modelo, $y_{t}$ é a variável dependente observada em um tempo $\mathrm{t} ; x_{t}(p+1)$ e $z_{t}(q+1)$ são vetores de covariância e $\beta$ e $\delta_{j}(\mathrm{j}=1, \ldots, \mathrm{m}+1)$ são os vetores dos coeficientes; $u_{t}$ é um distúrbio no tempo t. As quebras estruturais $(\mathrm{T} 1, \ldots, \mathrm{Tm})$ são tratadas como desconhecidas, utilizando-se a convenção de que T0 $=0$ e $\mathrm{Tm}+1=\mathrm{T}$. Quando um dos $\beta$ 's não está sujeito a mudanças ao longo da amostra completa há quebra parcial. Quando $\mathrm{p}=0$ acontece uma quebra estrutural pura, neste caso, todos os coeficientes dos regressores são alterados. A variância do erro $u_{t}$ não precisa ser constante, desde que as quebras de variância sejam coincidentes com as quebras nos parâmetros da regressão (BAI; PERRON, 2003). A equação (1) pode ser expressa em forma de matriz da seguinte forma:

$$
Y=X \beta+\bar{Z} \delta+U
$$

em que $Y=\left(y_{1}, \ldots, y_{T}\right)^{\prime}, X=\left(x_{1}, \ldots, x_{T}\right)^{\prime}, U=\left(\mathrm{u}, \ldots, u y_{T}\right)^{\prime}, \delta=\left(\delta_{1}^{\prime}, \delta_{2}^{\prime}, \ldots, \delta_{m+1}^{\prime}\right)^{\prime}$ e $\bar{Z}$ é a matriz com diagonal $\mathrm{Z}$ no $\mathrm{T} 1, \ldots$, Tm. Para cada m quebra $(\mathrm{T} 1, \ldots, \mathrm{Tm})$ uma regressão por Mínimos Quadrados Ordinários (MQO) associada é obtida pela minimização dos quadrados dos resíduos (equação 3).

$$
(Y-X \beta-\bar{Z} \delta)^{y}(Y-X \beta-\bar{Z} \delta)=\sum_{i=1}^{m+1} \sum_{t=T_{i-1}+1}^{T i}\left[y_{t}-x_{t}^{y} \beta-z_{t}^{y} \delta_{i}\right]^{2}
$$

na qual $\hat{\beta}\left[\left\{T_{j}\right\}\right]$ e $\hat{\delta}\left[\left\{T_{j}\right\}\right]$ representam as estimativas baseadas em $m$ quebras $\left(T_{1}, \ldots, T_{m}\right)$ denotadas por $\left\{T_{j}\right\}$. Substituindo estes na função objetiva e denotando a soma resultante do quadrado dos resíduos como $S_{T}\left(T_{1}, \ldots, T_{m}\right)$, as quebras estimadas são $\left(\widehat{T}_{1}, \ldots, \widehat{T}_{m}\right)$ é tal que $\left(\widehat{T}_{1}, \ldots, \widehat{T}_{m}\right)=\operatorname{argmin}_{T_{1, \ldots m} T_{m}} S_{t}\left(T_{1}, \ldots, T_{m}\right)$ na qual a minimização é considerada em todas as quebras $\left(T_{1}, \ldots, T_{m}\right)$ como $T_{i}-T_{i-1} \geq q^{2}$. Os parâmetros da regressão são estimados de forma associada às $m$ quebras $\left\{T_{j}\right\}$, como que $\hat{\beta}=\hat{\beta}\left[\left\{T_{j}\right\}\right]$ e $\hat{\delta}=\hat{\delta}\left[\left\{T_{j}\right\}\right]$. As quebras são testadas sequencialmente iniciando-se o teste entre 0 versus 1, em seguida, 1 versus 2 quebras e avançando até o que as $m$ quebras ( $m$ versus $m+1$ quebras) atinjam o ponto ótimo, no qual minimiza-se a soma dos quadrados dos resíduos (SSR).

Os intervalos entre quebras são chamados de regimes $(j)$. Portanto, para cada quebra estrutural será somado 1 para saber o número de regimes. Por exemplo, quando existe 1 quebra estrutural, há 2 dois regimes, um antes e outro após a quebra. Quando existem $m$ quebras, 
existem $m+1$ regimes $(j=m+1)$. Na seção seguinte é apresentado o modelo empírico do artigo.

\subsection{Modelo empírico e fonte dos dados}

O modelo baseia-se na premissa de que variáveis setoriais e variáveis sistêmicas tem impacto nas exportações de certo produto. Neste caso, o produto é a carne suína, incluindo as formas resfriada e congelada, além dos diferentes cortes e miúdos e, ainda, a carne processada. Empiricamente, pode ser expresso pela equação (4):

$$
E X_{-} S U_{t j}=\beta_{0}+\beta_{1} P I B_{t j}+\beta_{2} I N F+\beta_{3} T X_{-} J_{t j}+\beta_{4} E X_{-} M I_{t j}+\beta_{5} A B_{-} S U_{3}+u_{t j}
$$

em que:

$E X \_S U$ : quantidade exportada de suínos em mil toneladas por trimestre (USDA/ERS, 2015); $P I B$ : PIB trimestral (OECD, 2015);

$I N F$ : inflação representada pelo Producer Price Index Industry Data (PPI) (OECD, 2015);

$T X \_J$ : taxa de juros (OECD, 2015);

EX_MI : exportações de milho em mil toneladas (USDA/ERS, 2015);

$A B \_S U$ : abates de suínos em mil cabeças (USDA/ERS, 2015);

$u$ : o termo de erro aleatório com distribuição normal, isto é, média zero e variância constante;

$t$ : período de tempo em trimestres;

$j:$ regimes de quebras estruturais.

Na seção seguinte são apresentados os resultados dos testes e estimação do modelo.

\section{RESULTADOS}

Os resultados estão estruturados em três subseções. Na primeira são apresentadas as estatísticas descrivas das séries. Na segunda, os testes das propriedades das séries. Na terceira, o modelo empírido com quebras estruturais.

Nos gráficos das séries é possível observar constante crescimento nas exportações de carnes suínas até 2008, quando a crise mundial fez com que as exportações sofressem queda. O mesmo movimento pode ser observado na série do Produto Interno Bruto e na inflação. Nos Estados Unidos, as taxas de juros reais da economia mantiveram-se negativas no período analisado. As exportações de milho, principal componente da alimentação dos animais é irregular, apresentando picos e baixas durante o período. Os abates também mantiveram uma tendência de crescimento no período analisado (Figura 3). 
Exportação de Suínos (EX_SU)

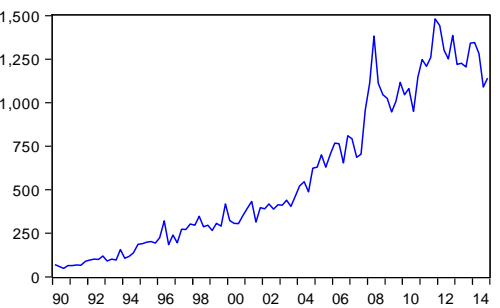

Taxa de Juros (TX J)

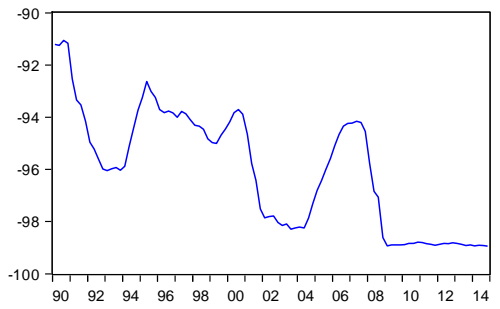

Fonte: Dados da pesquisa.
PIB

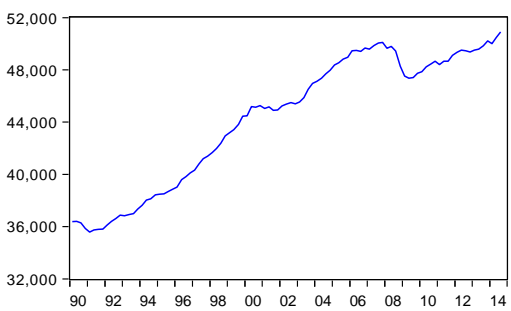

Exportação milho (EX_MI)

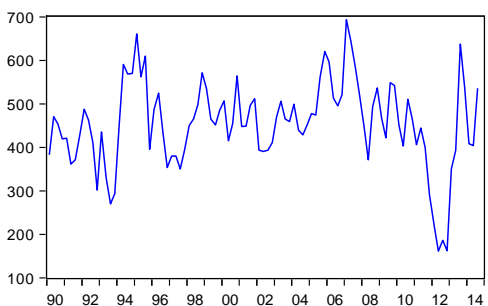

Inflação (INF)

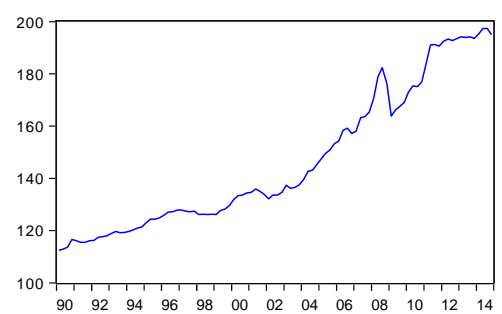

Abate de Suinos (AB_SU)

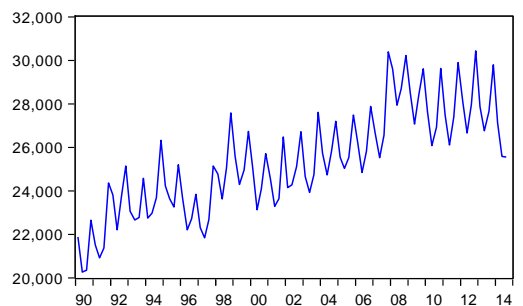

Figura 3 - Evolução das séries temporais coletadas dos Estados Unidos no período de 1990 a 2014, em mil toneladas

O teste de Dickey-Fuller Aumentado (DICKEY; FULLER, 1979) feito nas séries em nível e em primeira diferença mostra que somente a exportação do milho é estacionária em nível, mas também é em primeira diferença. Quando as séries são estacionárias em primeira diferença é possível checar se existem vetores de cointegração. Para isto foi utilizado o teste de Engle e Granger (1987) que apontou relação de cointegração entre algumas das séries (Tabela 2).

Tabela 2 - Teste de raíz unitária de Dickey-Fuller Aumentado (ADF) das séries em nível e em primeira diferença e teste de cointegração de Engle-Granger

\begin{tabular}{|c|c|c|c|c|c|c|c|c|}
\hline \multirow{3}{*}{ Variáveis } & \multicolumn{4}{|c|}{ Augmented Dickey-Fuller } & \multirow{2}{*}{\multicolumn{4}{|c|}{ Engle-Granger cointegration test }} \\
\hline & \multicolumn{2}{|c|}{ Em nível } & \multicolumn{2}{|c|}{ Primeira diferença } & & & & \\
\hline & t-Statistic & Prob.* & t-Statistic & Prob.* & $\begin{array}{l}\text { tau- } \\
\text { statistic }\end{array}$ & Prob.** & z-statistic & Prob.** \\
\hline$\overline{\text { EX_SU }}$ & -0.8960 & 0.7859 & -11.4317 & 0.0000 & -7.7879 & 0.0000 & -131.8048 & 0.0000 \\
\hline INF & 0.5569 & 0.9878 & -9.5684 & 0.0000 & -6.6630 & 0.0003 & -67.6622 & 0.0000 \\
\hline PIB & -0.9610 & 0.7645 & -6.3706 & 0.0000 & -1.4853 & 0.9951 & -4.6372 & 0.9979 \\
\hline TX_J & -2.4370 & 0.1344 & -4.6125 & 0.0003 & -4.2013 & 0.1958 & -80.4031 & 0.0000 \\
\hline EX_MI & -3.9990 & 0.0021 & -9.8367 & 0.0000 & -5.1286 & 0.0277 & -41.9067 & 0.0233 \\
\hline AB_SU & -1.5037 & 0.5274 & -5.4330 & 0.0000 & -4.2538 & 0.1809 & 48.2655 & 1.0000 \\
\hline
\end{tabular}

*MacKinnon (1996) one-sided p-values. **MacKinnon (1996) p-values.

Fonte: Dados da Pesquisa.

Em seguida são apresentadas a regressão com quebras estruturais, para explicar as relações de longo prazo entre as variáveis, e a regressão com vetor de cointegração, para as 
relações de curto prazo. O teste de Bai e Perron $(1998,2003)$ foi aplicado resultando em duas quebras estruturais, conforme tabela 3.

\begin{tabular}{cccc} 
Tabela 3 - Teste de múltiplas quebras estruturais \\
\hline Break Test & F-statistic & $\begin{array}{c}\text { Scaled } \\
\text { F-statistic }\end{array}$ & $\begin{array}{c}\text { Critical } \\
\text { Value** }\end{array}$ \\
\hline 0 vs. $1 *$ & $\mathbf{5 5 . 0 9 0 9}$ & $\mathbf{3 3 0 . 5 4 5 4}$ & $\mathbf{2 0 . 0 8 0 0}$ \\
$\mathbf{1}$ vs. $\mathbf{2} *$ & $\mathbf{9 . 8 0 8 7}$ & $\mathbf{5 8 . 8 5 2 0}$ & $\mathbf{2 2 . 1 1 0 0}$ \\
2 vs. 3 & 3.3935 & 20.3608 & 23.0400 \\
3 vs. 4 & 2.7333 & 16.4000 & 23.7700 \\
4 vs. 5 & 0.0000 & 0.0000 & 24.4300
\end{tabular}

Estimated break dates:

1: $2008 \mathrm{Q} 2$

2: 2000Q1, 2008Q4

3: 2000Q1, 2004Q4, 2008Q3

4: 1996Q2, 2000Q1, 2004Q4, 2008Q3

5: 1996Q2, 2000Q1, 2003Q3, 2007Q1, 2010Q3

* Significant at the 0.05 level

** Bai-Perron (2003) critical values.

Fonte: Dados da pesquisa

Considerando a existência de duas quebras, a regressão das relações de longo prazo pode ser vista na tabela 4, com duas quebras e, consequentemente, três regimes. As variáveis da regressão referem-se sempre ao trimestre anterior ao da variável dependente $(\mathrm{t}-1)$, assim, interpreta-se que o as mudanças nas variáveis independentes têm um impacto nas exportações do trimestre seguinte. Justifica-se pois o ciclo operacional do suíno é de 150 a 168 dias (MIELE, 2006), assim, existe uma demora no ajuste das exportações da carne suína em relação às mudanças setoriais e sistêmicas. Adotou-se o nível de significância de 5\% para a análise.

O primeiro regime $(j=1)$ refere-se ao período de 1990 a 1999. Neste regime, foram significativos os impactos da inflação e do PIB dos Estados Unidos nas exportações de carne suína. A direção dos sinais mostra que aumentos nas duas variáveis fizeram com que as exportações também aumentassem. Para os Estados Unidos, a década de 1990 foi um período de mudança na estrutura produtiva, enquanto a produção de suínos crescia a uma média de $1 \%$ ao ano, o número de produtores decrescia a $7 \%$ ao ano, sugerindo que o país estava se especializando, os pequenos produtores estavam saindo do negócio, e o resultado foram fazendas produtoras com três vezes mais animais no início do ano 2000. Dessa forma, a economia gerada pela produção em escala e especialização foram os responsáveis pelo bom resultado do período (OECD, 2003). 
O segundo regime $(j=2)$ refere-se ao período de 2000 a 2008 (terceiro trimestre). Novamente a inflação teve impacto positivo nas exportações. A taxa de juros dos Estados Unidos exerceu influência negativa no semestre seguinte de exportações de carne suína, assim, a medida que os juros diminuíram, as vendas para o exterior cresceram. É interessante observar que os abates de suínos em um trimestre, no segundo regime, tiveram impacto negativo nas exportações, não havendo uma justificativa teórica para tal. Pode-se conjecturar que este período foi de expansão do mercado da carne suína, assim, os Estados Unidos podem ter-se beneficiado. Próximo ao nível de 5\%, o PIB teve impacto negativo nas exportações das carnes suínas neste regime. Neste regime os contratos de produção foram amplamente difundidos nos Estados Unidos e geraram ganhos de eficiência e escala produtiva, conferindo ao país grande competitividade no mercado internacional (KEY; MCBRIDE, 2007; LOWE; GEREFFI, 2008).

O terceiro regime $(j=3)$ refere-se ao período de 2008 (quarto trimestre) até 2014 (terceiro trimestre). Foi um período no qual as exportações ficaram mais sensíveis a variações em todas as variáveis selecionadas, exceto o PIB. Neste período pós-crise mundial, a inflação interna dos EUA afetando positivamente as exportações. A taxa de juros afetou negativamente as exportações de carne suína, assim, maiores juros fizeram as vendas diminuírem. As exportações de milho também impactaram negativamente, mais milho no mercado interno pode ter incentivado a produção de carnes para exportação com maior valor agregado. Apesar de ter apresentado bons resultados, os Estados Unidos, passaram por duas crises no período. A primeira, provocada pelo surto de "gripe suína", que mais tarde, após perceberem o impacto na suinocultura, as autoridades passaram a chamar a doença de gripe $\mathrm{H} 1 \mathrm{~N} 1$, no entanto, diversos países (China, Rússia e Coréia do Sul) fizeram restrição total ou parcial à carne suína estadunidense (JOHNSON, 2009). A segunda em 2014, quando a Rússia voltou a embargar os produtos estadunidense.

Tabela 4 - Regressão com múltiplas quebras estruturais

\begin{tabular}{ccccc}
\hline Variáveis & Coeficientes & Erro Patrão & t-Statistic & Prob. \\
\hline C & $\mathbf{1 9 9 0 Q 1}-\mathbf{1 9 9 9 Q 4}-\mathbf{4 0}$ obs $(\boldsymbol{j}=\mathbf{1})$ & & \\
PIB(1) & $\mathbf{- 1 5 4 0 . 2 7 3 0}$ & $\mathbf{2 4 3 . 2 1 9 3}$ & $\mathbf{- 6 . 3 3 2 9}$ & $\mathbf{0 . 0 0 0 0}$ \\
INF(1) & $\mathbf{0 . 0 2 2 1}$ & $\mathbf{0 . 0 0 4 9}$ & $\mathbf{4 . 5 2 0 1}$ & $\mathbf{0 . 0 0 0 0}$ \\
TX_J(1) & $\mathbf{7 . 1 8 1 1}$ & $\mathbf{2 . 1 5 5 7}$ & $\mathbf{3 . 3 3 1 3}$ & $\mathbf{0 . 0 0 1 3}$ \\
EX_MI(1) & -0.2068 & 2.8755 & -0.0719 & 0.9429 \\
AB_SU(1) & 0.0513 & 0.0612 & 0.8385 & 0.4042 \\
& -0.0026 & 0.0031 & -0.8377 & 0.4047 \\
C & $\mathbf{2 0 0 0 Q 1}-\mathbf{2 0 0 8 Q 3 - - 3 5}$ obs $(j=2)$ & & \\
PIB(1) & $\mathbf{- 3 5 8 9 . 3 6 6 0}$ & $\mathbf{3 7 6 . 8 7 8 7}$ & $\mathbf{- 9 . 5 2 3 9}$ & $\mathbf{0 . 0 0 0 0}$ \\
\hline
\end{tabular}




\begin{tabular}{ccccc} 
INF(1) & $\mathbf{2 1 . 6 0 6 2}$ & $\mathbf{1 . 4 9 5 3}$ & $\mathbf{1 4 . 4 4 9 5}$ & $\mathbf{0 . 0 0 0 0}$ \\
TX_J(1) & $\mathbf{- 2 5 . 9 0 8 3}$ & $\mathbf{5 . 3 0 8 4}$ & $\mathbf{- 4 . 8 8 0 6}$ & $\mathbf{0 . 0 0 0 0}$ \\
EX_MI(1) & 0.1055 & 0.1373 & 0.7687 & 0.4443 \\
AB_SU(1) & $\mathbf{- 0 . 0 2 3 8}$ & $\mathbf{0 . 0 0 6 0}$ & $\mathbf{- 3 . 9 6 5 0}$ & $\mathbf{0 . 0 0 0 2}$ \\
& $\mathbf{2 0 0 8 Q 4}-\mathbf{2 0 1 4 Q 2}-\mathbf{2 3}$ obs $(\boldsymbol{j}=\mathbf{3})$ & & $\mathbf{- 4 . 2 4 7 4}$ & $\mathbf{0 . 0 0 0 1}$ \\
C & $\mathbf{- 8 1 3 6 5 . 6 9 0 0}$ & $\mathbf{1 9 1 5 6 . 5 9 0 0}$ & $\mathbf{- 4 0 . 1 6 4 4}$ & 0.8698 \\
PIB(1) & -0.0039 & 0.0239 & $\mathbf{0 . 1 3 2 4}$ & $\mathbf{0 . 0 0 0 1}$ \\
INF(1) & $\mathbf{9 . 1 3 6 4}$ & $\mathbf{2 . 2 1 0 9}$ & $\mathbf{- 4 . 1 8 4 5}$ & $\mathbf{0 . 0 0 0 1}$ \\
TX_J(1) & $\mathbf{- 8 2 6 . 1 2 6 4}$ & $\mathbf{1 9 7 . 4 2 6 0}$ & $\mathbf{- 4 . 3 1 1 5}$ & $\mathbf{0 . 0 0 0 0}$ \\
EX_MI(1) & $\mathbf{- 0 . 5 1 6 0}$ & $\mathbf{0 . 1 1 9 7}$ & $\mathbf{- 2 . 0 0 0 2}$ & $\mathbf{0 . 0 4 8 9}$ \\
AB_SU(1) & $\mathbf{- 0 . 0 1 4 6}$ & $\mathbf{0 . 0 0 7 3}$ & 566.9632 \\
R-squared & 0.9899 & Mean dependent var & 433.8241 \\
Adjusted R-squared & 0.9877 & S.D. dependent var & 10.7492 \\
S.E. of regression & 48.1089 & Akaike info criterion & 11.2240 \\
Sum squared resid & 185157.6000 & Schwarz criterion & 10.9413 \\
Log likelihood & -508.7117 & Hannan-Quinn criter. & 2.3351 \\
F-statistic & 459.2735 & Durbin-Watson stat & \\
Prob(F-statistic) & 0.0000 & & & \\
\hline
\end{tabular}

Nota: Parâmetros utilizados na regressão Sample (adjusted): 1990Q1 2014Q2, Included observations: 98 after adjustments, Break type: Bai-Perron tests of L+1 vs. L globally determined breaks, Break selection: Sequential evaluation, Trimming 0.15, Max. breaks 5, Sig. level 0.05, Breaks: 2000Q1, 2008Q4, HAC standard errors \& covariance (Bartlett kernel, Newey-West fixed bandwidth), Allow heterogeneous error distributions across breaks

Fonte: Dados da pesquisa.

Os resultados da regressão com múltiplas quebras estruturais geraram um $R^{2}$ ajustado de 0,9877, conferindo um bom ajuste do modelo. Os critérios de informação de Akaike, Hannan-Quinn e Schwarz foram baixos. O teste ADF aplicado nos resíduos da regressão confirmou a existência de cointegração entre as séries, pois, os resíduos são estacionários $(t=$ $-11.5527, p=0.0000)$.

\section{CONSIDERAÇÕES FINAIS}

Os Estados Unidos tiveram contínuo aumento das exportações de carnes suínas entre 1990 e 2014. O país é o maior exportador mundial destes produtos. O objetivo do artigo foi verificar alguns dos determinantes para este desempenho, utilizando quebras estruturais para localizar mudanças nos impactos destes determinantes.

Como resultado, duas quebras estruturais foram localizadas e diferentes impactos foram observados em cada um dos três regimes resultantes. Um primeiro período com maior impacto de variáveis sistêmicas (PIB e inflação), no qual o país buscava ganhos de eficiência produtiva e maior competitividade no mercado. Um segundo período com maior impacto do PIB, inflação, taxa de juros e abates de suínos, já com a eficiência garantida por um sistema de contratos com coordenação vertical intensa. Um terceiro período mais sensível às variáveis, tanto setoriais quanto sistêmicas, resultante da crise de 2008 além dos surtos de H1N1. 
A contribuição do artigo está no fato de compreender que as relações dos fatores sistêmicos e setoriais tem diferentes impactos em diferentes momentos do tempo, dando um caráter dinâmico à análise. Sugere-se, contudo, que novas pesquisas sejam realizadas com diferentes métodos para reforçar os resultados.

\section{AGRADECIMENTOS}

A pesquisa foi financiada pela Fundação de Amparo à Pesquisa de Mato Grosso (FAPEMAT). Contudo, a análise e os comentários são de responsabilidade dos autores.

\section{REFERÊNCIAS}

BAI, J.; PERRON, P. Estimating and Testing Linear Models with Multiple Structural Changes. Econometrica, v. 66, n. 1, p. 47-78, 1998. Disponível em: <http://www.jstor.org/stable/2998540>.

Computation and analysis of multiple structural change models. Journal of Applied Econometrics, v. 18, n. 1, p. 1-22, jan. 2003. Disponível em:

<http://doi.wiley.com/10.1002/jae.659>. Acesso em: 25 maio. 2014.

BATALHA, M. O.; SOUZA FILHO, H. M. Analisando a competitividade de cadeias agroindustriais: uma proposição metodológica. In: BATALHA, M. O.; SOUZA FILHO, H. M. (Org.). . Agronegócio no Mercosul: uma agenda para o desenvolvimento. São Paulo: Atlas, 2009. p. 1-22.

DICKEY, D. A.; FULLER, W. A. Distribution of the Estimators for Autoregressive Time Series With a Unit Root. Journal of the American Statistical Association, v. 74, p. 427431, 1979.

ENGLE, R. F.; GRANGER, C. W. J. Co-integration and error correction: representation, estimation, and testing. Econometrica, v. 55, p. 251-276, 1987. Disponível em: <http://www.jstor.org/discover/10.2307/1913236?uid=3738128\&uid=2\&uid=4\&sid=2110339 $7488771>$.

FAO. FAOSTAT: Food and Agriculture Organization of the United Nations Statistic Division. Disponível em: <http://faostat3.fao.org/>. Acesso em: 16 abr. 2015.

GEREFFI, G.; HUMPHREY, J.; STURGEON, T. J. The governance of global value chains. Review of International Political Economy, v. 12, n. 1, p. 78-104, fev. 2005. Disponível em: <http://dx.doi.org/10.1080/09692290500049805>. Acesso em: 23 maio. 2014.

GERVASIO, E. W. Suinocultura - Análise da Conjuntura Agropecuária: SEAB Secretaria de Estado da Agricultura e do Abastecimento do Paraná. Disponível em: $<$ http://www.agricultura.pr.gov.br/arquivos/File/deral/Prognosticos/SuinoCultura_2012_2013. pdf>. Acesso em: 14 out. 2010.

JOHNSON, R. Potential farm sector effects of 2009 H1N1 "swine flu": questions and answers. Disponível em: <https://www.fas.org/sgp/crs/misc/R40575.pdf>. Acesso em: 25 ago. 2008.

KEY, N.; MCBRIDE, W. The Changing Economics of U.S. Hog Production. Production, n. $52,2007$.

LOWE, M.; GEREFFI, G. A Value Chain Analysis of the U.S. pork industry. Disponível 
em:

$<$ http://www.cggc.duke.edu/environment/valuechainanalysis/CGGC_PorkIndustryReport_103-08.pdf >. Acesso em: 1 set. 2014.

MACKINNON, J. G. Numerical distribution functions for unit roor and cointegration tests. Journal of Applied Econometrics, v. 11, n. 6, p. 601-618, 1996.

MIELE, M. Contratos, especialização, escala de produção e potencial poluidor na suinocultura de Santa Catarina. Tese (Doutorado em Agronegócios) -- Universidade Federal do Rio Grande do Sul, 2006.

OECD. Agriculture, trade and the environment -the pig sector. Paris: OECD Publishing, 2003.

Organisation for Economic Co-operation and Development: Data. Disponível em: <https://data.oecd.org/>. Acesso em: 26 ago. 2015.

USDA. Global Agricultural Trade System online. Disponível em:

<http://apps.fas.usda.gov/gats/ExpressQuery1.aspx>. Acesso em: 1 maio. 2015a.

Production, supply and distribution online. Disponível em:

<http://apps.fas.usda.gov/psdonline/psdQuery.aspx>. Acesso em: 20 maio. 2003b.

USDA/ERS. United States Department of Agriculture: Economic Research Service.

Disponível em: <http://www.ers.usda.gov/>. Acesso em: 26 ago. 2015. 